\title{
Impact of Bimodal Traffic on Latency in Optical Burst Switching Networks
}

\author{
Yuhua Chen ${ }^{1}$ and Pramode K. Verma ${ }^{2}$ \\ ${ }^{1}$ Department of Electrical and Computer Engineering, University of Houston, Houston, TX 77204, USA \\ ${ }^{2}$ Telecommunications Engineering Program, School of Electrical and Computer Engineering, \\ The University of Oklahoma, Tulsa, OK 74135, USA
}

Correspondence should be addressed to Yuhua Chen, yuhua.chen@mail.uh.edu

Received 25 September 2008; Accepted 28 November 2008

Recommended by Luca De Nardis

This paper analyzes the impact of bimodal traffic composition on latency in optical burst switching networks. In particular, it studies the performance degradation to short-length packets caused by longer packets, both of which are part of a heterogeneous traffic model. The paper defines a customer satisfaction index for each of the classes of traffic, and a composite satisfaction index. The impact of higher overall utilization of the network as well as that of the ratio of the traffic mix on each of the customer satisfaction indices is specifically addressed.

Copyright (C) 2008 Y. Chen and P. K. Verma. This is an open access article distributed under the Creative Commons Attribution License, which permits unrestricted use, distribution, and reproduction in any medium, provided the original work is properly cited.

\section{Introduction}

Optical transmission technologies have been widely adopted in the core telecommunication network today. The adoption of optical transmission technology has been far more pervasive than the switching technology. Optical burst switching $[1,2]$ effectively fills the gap between coarse lambda switching and the still-distant optical packet switching [3, 4], and is considered the most promising form of optical switching technology [5].

Optical burst switching relies on relatively large bursts of data assembled at the ingress node to be transported over an end-to-end optical path to the destination or egress node. Since it is economically infeasible to form a burst in response to each customer packet, the packets entering the ingress node will likely originate from different sources and thus have varying statistical characteristics. In this paper, we consider this traffic to have a bimodal distribution, each component of which is negative exponentially distributed with a separate mean. This assumption is justifiable in the instance when the ingress traffic is a mixture of short packets and relatively long packets, for example, packets containing dense payloads that allow sharing of resources of a grid network to a remote point [6].
It is generally known that heterogeneous traffic leads to poor performance, specifically, as far as the smaller-length traffic is concerned. However, it is imperative from a service provider point of view to quantify and understand the impact of the heterogeneous traffic on the network as a whole as well as on each of the constituent customer groups. In this paper, we introduce the concept of a customer satisfaction index for each of the two classes of traffic, and a composite customer satisfaction index for the network as a whole. We then derive a closed form mathematical solution for the individual and composite customer satisfaction indices as a function of the ratio of the mean lengths and further as a function of the overall traffic intensity.

\section{Bimodal Traffic Model}

In this paper, we are interested in evaluating the impact of the bimodal traffic on the latency of the two traffic types, as well as on the system latency as a whole. More specifically, we want to understand how the mixed traffic affects the latency experienced by individual traffic type. We define the individual customer satisfaction index as follows: $\eta_{1}=T_{1} / T_{c}$, and $\eta_{2}=T_{2} / T_{c}$, where $T_{1}$ and $T_{2}$ are the latency experienced by type 1 an type 2 traffic in systems carrying single type of 
traffic, respectively, and $T_{c}$ is the latency experienced by the composite traffic. We further define the composite customer satisfaction index as $\eta=\delta \cdot \eta_{1}+(1-\delta) \cdot \eta_{2}$, where $\delta(0 \leq \delta \leq$ 1 ) is the portion of traffic type 1 in the composite traffic load $\rho_{c}$.

In the rest of the section, we derive a mathematical model for the individual and composite customer satisfaction indices in terms of the composite load and the ratio of the mean message lengths of the two traffic types.

We assume that each component of the bimodal traffic follows a Poisson arrival process. Since the message lengths of the two streams are negative exponential distributed, we have the distribution of the composite stream as

$$
f(x)=\frac{\lambda_{1}}{\lambda_{c}} \mu_{1} e^{-\mu_{1} x}+\frac{\lambda_{2}}{\lambda_{c}} \mu_{2} e^{-\mu_{2} x} .
$$

Equation (1) follows from the fact that the probability density function of the composite stream is the sum of the density functions of the two streams weighted by the relative probabilities of their occurrence. Note that the combined stream is not negative exponentially distributed unless $\mu_{1}=$ $\mu_{2}$.

We derive the average latency of the composite stream as follows. For evaluating the mean delay suffered by an individual message, we have [7]

$$
T=\frac{1}{\mu C}+\frac{\rho^{2}+\lambda^{2} \sigma_{S}^{2}}{2 \lambda(1-\rho)}
$$

where $1 / \mu$ is the mean message length, $\sigma_{S}^{2}$ is the variance of the service time and $C$ is the channel rate at which the messages are being emptied. Equation (2) holds for any message length distribution. Let $\sigma_{c}^{2}$ be the variance of the message length distribution.

For the combined stream, the mean length can be evaluated as

$$
\frac{1}{\mu_{c}}=\overline{x_{c}}=\int_{0}^{\infty} x f(x) d x=\frac{1}{\lambda_{c}} \cdot\left(\frac{\lambda_{1}}{\mu_{1}}+\frac{\lambda_{2}}{\mu_{2}}\right) .
$$

Let $g_{1}(x)=\int_{0}^{\infty} \mu_{1} e^{-\mu_{1} x} d x$ and $g_{2}(x)=\int_{0}^{\infty} \mu_{2} e^{-\mu_{2} x} d x$. The Laplace transform of $g_{1}(x)$ is $G_{1}(s)=\mu_{1} /\left(\mu_{1}+s\right)$. The second moment of $g_{1}(x)$ can be calculated using

$$
\overline{x_{g_{1}}^{2}}=\left.\frac{d^{2} G_{1}^{*}(s)}{d s^{2}}\right|_{s=0}=\left.\frac{d^{2}}{d s^{2}}\left(\frac{\mu_{1}}{\mu_{1}+s}\right)\right|_{s=0}=\frac{2}{\mu_{1}^{2}} .
$$

We also have $\overline{g_{2}^{2}}=2 / \mu_{2}{ }^{2}$. The second moment of the combined stream can be calculated as

$$
\begin{aligned}
\overline{x_{c}^{2}} & =\int_{0}^{\infty} x^{2} f(x) d x \\
& =\frac{\lambda_{1}}{\lambda_{c}} \overline{x_{1}^{2}}+\frac{\lambda_{2}}{\lambda_{c}} \overline{x_{2}^{2}}=\frac{2}{\lambda_{c}}\left(\frac{\lambda_{1}}{\mu_{1}{ }^{2}}+\frac{\lambda_{2}}{\mu_{2}{ }^{2}}\right) .
\end{aligned}
$$

The variance of the combined stream can be derived as

$$
\begin{aligned}
\sigma_{c}^{2} & =\overline{x_{c}^{2}}-\left(\overline{x_{c}}\right)^{2} \\
& =\frac{2}{\lambda_{c}}\left(\frac{\lambda_{1}}{\mu_{1}{ }^{2}}+\frac{\lambda_{2}}{\mu_{2}{ }^{2}}\right)-\frac{1}{\lambda_{c}{ }^{2}} \cdot\left(\frac{\lambda_{1}}{\mu_{1}}+\frac{\lambda_{2}}{\mu_{2}}\right)^{2} .
\end{aligned}
$$

From (6) $\sigma_{S}^{2}$ can be calculated using $\sigma_{S}^{2}=\sigma_{c}^{2} / C^{2}$. We then define the ratio of type 2 message-length to type 1 messagelength as $\gamma=\mu_{1} / \mu_{2}$. Let $\chi=\delta+(1-\delta) / \gamma$. Equation (3) becomes

$$
\frac{1}{\mu_{c}}=\frac{1}{\mu_{1}} \cdot \frac{1}{\delta+(1-\delta) / \gamma}=\frac{1}{\chi \mu_{1}} .
$$

We can rewrite the variance of the service time of the combined stream as

$$
\sigma_{S}^{2}=\frac{1}{\mu_{1}^{2} C^{2}}\left(\frac{2(\delta+\gamma(1-\delta))}{\chi}-\frac{1}{\chi^{2}}\right) .
$$

Substituting (7) and (8) in (2), we have

$$
T=\frac{1}{\chi \mu_{1} C}\left(1+\frac{\rho_{c}(2 \chi(\delta+\gamma(1-\delta))-1)}{2 \chi^{2} \mu_{1}^{2} C^{2}\left(1-\rho_{c}\right)} \cdot\left(1+\chi^{2} \mu_{1}^{2} C^{2}\right)\right) .
$$

Using the mean service times of the messages from the two streams, we have

$$
T_{1}=\frac{1}{\mu_{1} C}+\frac{\rho_{1}}{\mu_{1} C\left(1-\rho_{1}\right)} .
$$

$T_{2}$ can be similarly calculated. Thus, we have the individual customer satisfaction index

$$
\begin{aligned}
& \eta_{1}=\chi\left(1+\frac{\rho_{c}(2 \chi(\delta+\gamma(1-\delta))-1)}{2 \chi^{2} \mu_{1}^{2} C^{2}\left(1-\rho_{c}\right)} \cdot\left(1+\chi^{2} \mu_{1}^{2} C^{2}\right)\right)^{-1}, \\
& \eta_{2}=\gamma \cdot \chi\left(1+\frac{\rho_{c}(2 \chi(\delta+\gamma(1-\delta))-1)}{2 \chi^{2} \mu_{1}^{2} C^{2}\left(1-\rho_{c}\right)} \cdot\left(1+\chi^{2} \mu_{1}^{2} C^{2}\right)\right)^{-1} .
\end{aligned}
$$

The composite customer satisfaction index $\eta$ can be calculated based on definition.

\section{Numerical Results}

Figures 1 through 3 present plots that capture the variations of the three different customer satisfaction indices as a function of the ratio of the two mean message lengths at various composite loads. The composite traffic, in each case, consists of $50 \%$ of the bits from the shorter and $50 \%$ bits from the longer messages.

As can be seen from Figure 1, with a composite load of 0.4 , the satisfaction index of the customer group generating the shorter messages rapidly declines as the ratio of the mean message lengths increases. This decline can be observed to be true for all levels of the composite load. The latter observation follows from Figures 2 and 3 which address higher levels of load. This is consistent with the fact that a preponderance of longer messages increasingly affects the satisfaction index of the shorter messages.

It can be observed from all the three figures taken together that all the customer satisfaction indices decline as a function of the composite load. The intersection of the three indices when the two customer groups generate statistically identical messages verifies the fact that satisfaction indices 


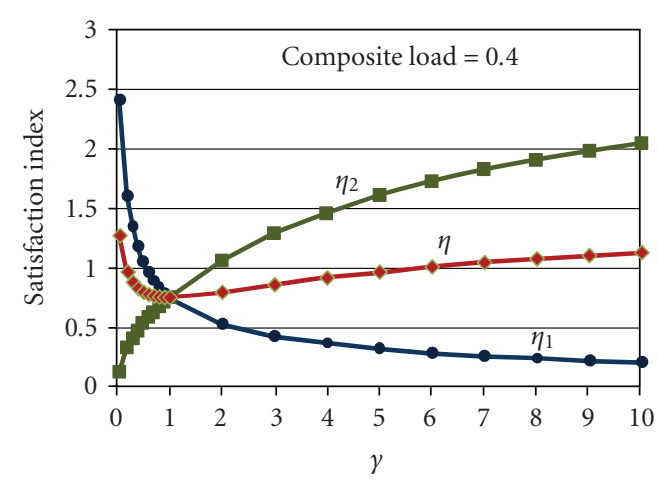

FIgURE 1: Customer satisfaction index at low load.

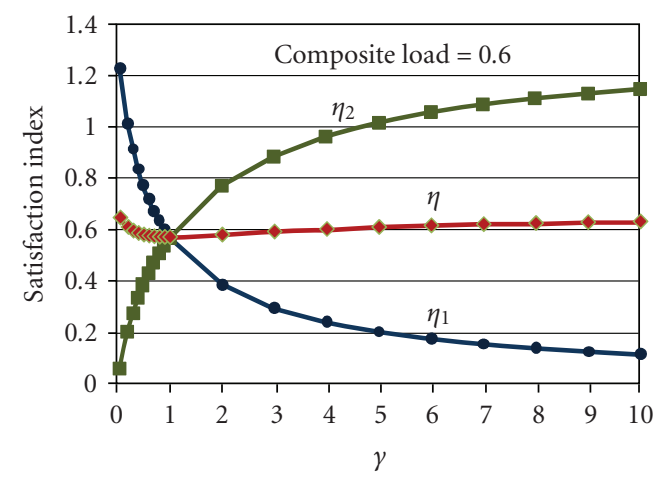

FIgURE 2: Customer satisfaction index at medium load.

cannot differ when the messages from the two groups are statistically identical.

Simultaneously, it can be observed that when the ratio of the mean lengths increases, the composite satisfaction index holds largely constant while the satisfaction index of the users of longer messages shows an increase. This is attributed to the fact that as the ratio of the message lengths increases, the mean latency in systems carrying only longer messages also increases, while longer messages in composite systems benefit from the existence of shorter messages, resulting in an increase in satisfaction indices.

\section{Conclusion}

This paper has defined parameters that reflect the satisfaction of customer groups generating bimodal traffic that constitutes the payload of the OBS burst. It has derived analytical results relating the customer satisfaction indices to variations in the ratio of the average message lengths of the two groups and to the increase in the overall utilization factor. The results clearly indicate the reducing satisfaction of all the indices as the load is increased, and especially that of the customer group generating shorter messages, as the ratio of the mean lengths of the larger to smaller messages is increased. However, surprisingly, the network as a whole, as indicated by the composite satisfaction index, is largely not affected by the ratio of the mean lengths due to the interplay

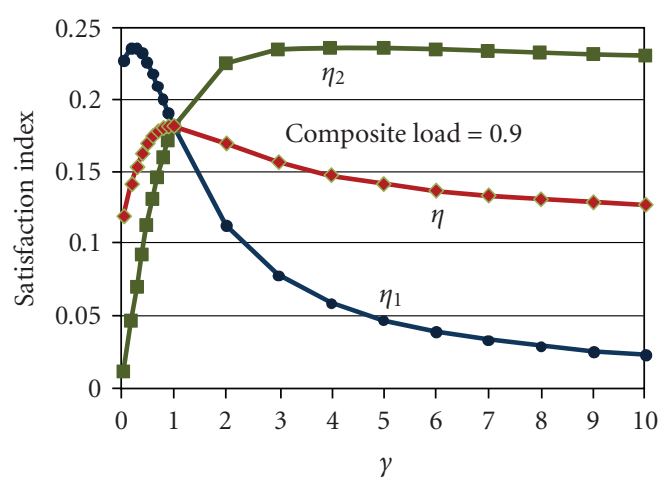

FIgURE 3: Customer satisfaction index at heavy load.

between the longer and shorter messages in the composite system.

\section{Acknowledgments}

This work was supported in part by the National Science Foundation (NSF) under Grant CNS-0708613, and the Texas Advanced Research Program (ARP) under Grant G096059.

\section{References}

[1] J. S. Turner, “Terabit burst switching," Journal of High Speed Networks, vol. 8, no. 1, pp. 3-16, 1999.

[2] C. Qiao and M. Yoo, "Optical burst switching (OBS)-a new paradigm for an optical internet," Journal of High Speed Networks, vol. 8, no. 1, pp. 69-84, 1999.

[3] D. J. Blumenthal, P. R. Prucnal, and J. R. Sauer, "Photonic packet switches: architectures and experimental implementations," Proceedings of the IEEE, vol. 82, no. 11, pp. 1650-1667, 1994.

[4] P. Gambini, M. Renaud, C. Guillemot, et al., "Transparent optical packet switching: network architecture and demonstrators in the KEOPS project," IEEE Journal on Selected Areas in Communications, vol. 16, no. 7, pp. 1245-1259, 1998.

[5] M. J. O'Mahony, C. Politi, D. Klonidis, R. Nejabati, and D. Simeonidou, "Future optical networks," Journal of Lightwave Technology, vol. 24, no. 12, pp. 4684-4696, 2006.

[6] Y. Chen, W. Tang, and P. K. Verma, "Latency in grid over optical burst switching with heterogeneous traffic," in Proceedings of the 3rd International Conference on High Performance Computing and Communications (HPCC '07), vol. 4782 of Lecture Notes in Computer Science, pp. 334-345, Springer, Houston, Tex, USA, September 2007.

[7] D. G. Kendall, "Some problems in the theory of queues," Journal of the Royal Statistical Society Series B, vol. 13, pp. 151-185, 1951. 

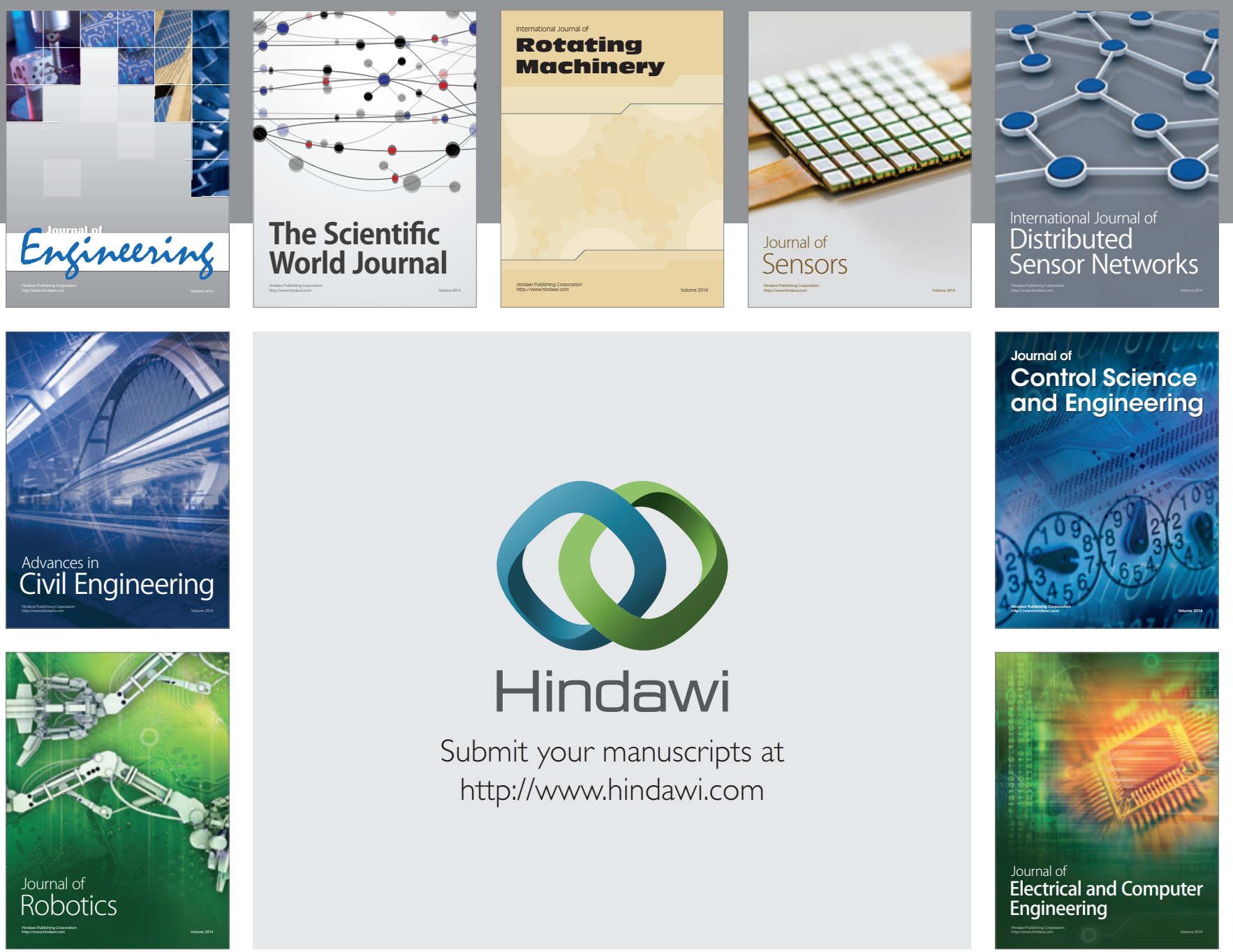

Submit your manuscripts at

http://www.hindawi.com
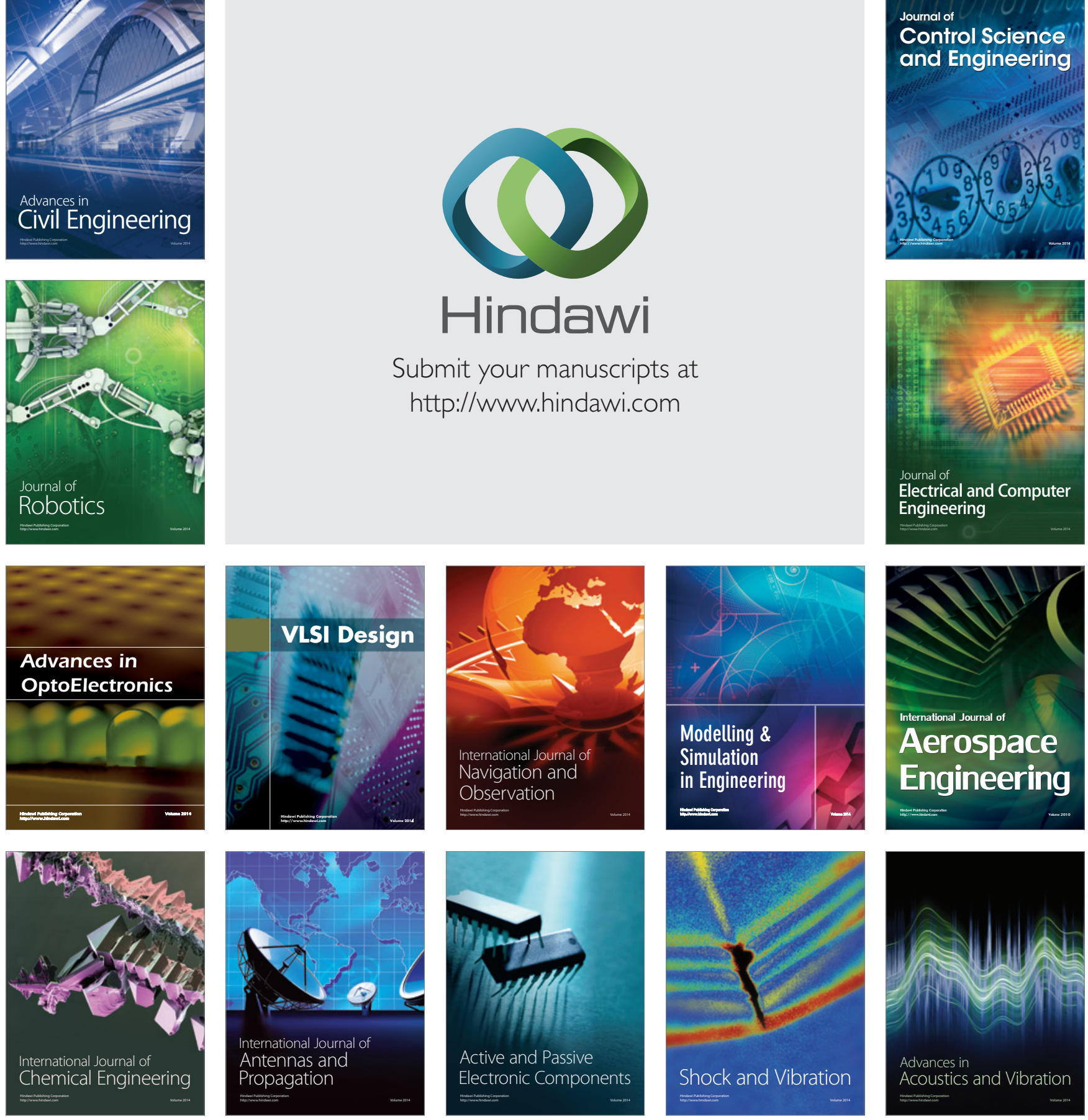